\title{
Downregulation of microRNA-15a suppresses the proliferation and invasion of renal cell carcinoma via direct targeting of eIF4E
}

\author{
GANG LI $^{1}$, TIE CHONG ${ }^{1}$, XIAOLONG XIANG ${ }^{1}$, JIE YANG $^{2}$ and HONGLIANG LI ${ }^{1}$ \\ ${ }^{1}$ Department of Urology, The Second Affiliated Hospital of Xi'an Jiaotong University, Xi'an, Shaanxi 710004; \\ ${ }^{2}$ Department of Nursing, Xi'an Beifang Chinese Medicine Skin Disease Hospital, Xi'an, Shaanxi 710002, P.R. China
}

Received April 28, 2017; Accepted July 26, 2017

DOI: 10.3892/or.2017.5901

\begin{abstract}
The downregulation of microRNA-15a has been reported in several human tumors. However, its expression and functional importance in renal cell carcinoma ( $\mathrm{RCC}$ ) remain unknown. The aim of the present study was to investigate its expression, biological functions and underlying mechanisms in RCC tumorigenesis. The expression levels of miR-15a were examined by qRT-PCR in 40 RCC specimens and adjacent-paired normal tissues. Cell Counting Kit-8 (CCK-8), colony formation, flow cytometry and Transwell assays were used to explore the potential influence of miR-15a transfection on RCC cell proliferation, the cell cycle, cell apoptosis, and cell invasion. Luciferase reporter assays were performed to confirm the potential target of miR-15a, in combination with qRT-PCR, western blotting and immunohistochemical assays . We found that miR-15a was significantly downregulated in most RCC specimens compared with adjacent normal tissues $(\mathrm{P}<0.01)$. Overexpression of miR-15a inhibited cellular growth, suppressed invasion and arrested cells at the G1/G0 phase, and induced cell apoptosis in RCC cells. Luciferase assays revealed that miR-15a directly targeted the binding site of the 3'-untranslated region (3'-UTR) of eIF4E, and inhibited its expression at both mRNA and protein levels. eIF4E expression was negatively associated with miR-15a expression in RCC tissues. eIF4E overexpression treatment partially abrogated the inhibitory effect of miR-15a on cell proliferation and invasion, as well as inactivated $\mathrm{P} 13 \mathrm{~K} / \mathrm{AKT} / \mathrm{mTOR}$ signaling in RCC cells. In conclusion, the present study indicated that miR-15a downregulation was associated with cell proliferation and invasion by directly targeting eIF4E during RCC progression. Thus, it may serve
\end{abstract}

Correspondence to: Dr Tie Chong, Department of Urology, The Second Affiliated Hospital of Xi'an Jiaotong University, 157 Xiwu Road, Xi'an, Shaanxi 710004, P.R. China

E-mail: tiechongery@163.com

Key words: miR-15a, eIF4E, renal cell carcinoma, proliferation, invasion as a potential tumor suppressor and therapeutic target for the treatment of RCC.

\section{Introduction}

Renal cell carcinoma ( $\mathrm{RCC}$ ) is the second most prevalent urologic malignancy in China only after bladder cancer (1). It often arises without early symptoms, thus $20-30 \%$ patients are diagnosed when distant metastases already present (2). Surgery is the current major therapeutic approach for RCC patients, whereas, for patients with advanced RCC, who are not suitable for surgery, or whose solitary kidney cannot be completely excised by surgery, radiation and chemotherapy can be applied as alternative treatments $(3,4)$. However, clinical investigations report that the sensitivity of RCC patients to radiation and chemotherapy is extremely low, greatly restricting the clinical therapeutic effects on advanced RCC patients (5). Thus, unraveling the molecular mechanisms underlying the initiation and progression of RCC may provide a better understanding of the therapy of RCC.

MicroRNAs (miRNAs) are usually 20-24 nucleotides in length and non-coding small RNAs that transcriptionally and post-transcriptionally mediate $60 \%$ of human protein coding gene expression and participate in a variety of biological processes including cell differentiation, proliferation and apoptosis $(6,7)$. miRNAs function as either oncogenes or tumor-suppressor genes by binding to complementary sequences at 3'-untranslated regions (3'-UTRs) of target messenger RNAs in diverse cancers $(8,9)$. Among them, Li et al have identified miR-21 as an oncogenic driver in RCC cells that regulates cell invasion (10). Xu et al have suggested that miR-203 could be a prognostic marker and serves as a tumor suppressor in human RCC cells (11). Recent studies have shown that downregulation of miR-15a is involved in the tumorigenesis and progression of several human types of cancer (12-14). However, the role that miR-15a plays in the carcinogenesis of RCC is still unclear.

Eukaryotic translation initiation factor 4E (eIF4E) as an mRNA cap-binding protein is regulated via phosphorylation by binding to eukaryotic initiation factor $4 \mathrm{E}$ binding proteins (4E-BPs) (15). It is the most efficient speed regulator for eukaryotic mRNA translation and plays an important regulatory role 
in the initial phase of protein synthesis (16). Overexpression of eIF4E causes preferential translation of mRNAs containing excessive secondary structures in their 5'-UTR that are normally inefficiently translated, such as growth promoting proteins and oncogenic proteins (17). Through this mechanism, eIF4E overexpression in cancer cells is associated with cancer-related events such as transformation, angiogenesis, invasion and metastasis (18). Accordingly, the aberrant expression of eIF4E is reported to be closely related to the occurrence and development of several tumors including RCC (19).

In the present study, the expression of miR-15a was evaluated in the RCC tissue specimens, and the functions of miR-15a and the mechanisms involved were also investigated. We demonstrated that miR-15a expression was significantly downregulated in RCC specimens when compared with that of adjacent normal tissues. Its overexpression inhibited proliferation and invasion of RCC cells, in association with blocking cell cycle progression and inducing cell apoptosis by directly targeting eIF4E. These data strongly demonstrated the tumor-suppressor role of miR-15a in the development of human RCC.

\section{Materials and methods}

Specimens. Fresh biopsy specimens of RCC and normal renal tissues from the incisal margin were collected from 40 patients with RCC who underwent radical surgery at The Second Affiliated Hospital of Xi'an Jiaotong University (Xian, China) from May 2011 to July 2012. None of the patients, aged 40-75 years (mean age, 58), had received any chemotherapy, radiotherapy or other adjuvant therapy before surgery. Informed consent was obtained from all patients, and the present study was approved by the Ethical Review Committee of Xi'an Jiaotong University and complied with the Declaration of Helsinki.

Cell culture and treatment. The human renal carcinoma cell lines (ACHN, 786-O, 769-P and OS-RC-2) and normal renal cell line HK-2 were obtained from the China Center for Type Culture Collection (CCTCC; Shanghai, China). The cells were cultured in Dulbecco's modified Eagle's medium (DMEM) supplemented with $10 \%$ (v/v) sterile newborn calf serum (NCBS) and antibiotics (10 U/ml penicillin and $10 \mu \mathrm{g} / \mathrm{ml}$ streptomycin). The cells were then incubated at $37^{\circ} \mathrm{C}$ in a humidified chamber supplemented with $5 \% \mathrm{CO}_{2}$. For transfections, miR-15a and negative control mimics, pcDNA3.1-eIF4E and negative control plasmids were synthesized by GenePharma (Shanghai, China) and transfected into 769-P and OS-RC-2 cells using Lipofectamine 2000 (Invitrogen, Carlsbad, CA, USA) according to the manufacturer's instructions.

Cell proliferation assay. Cells were transfected with miR-15a mimics or NC for $48 \mathrm{~h}$, and then $\sim 4 \times 10^{3}$ cells were plated into each well of a 96-well plate and incubated overnight. The medium was removed, and Cell Counting solution [Cell Counting Kit-8 (CCK-8); Beyotime, Jiangsu, China] was added to each well and incubated for $1 \mathrm{~h}$. The absorbance of solubilized dye was assessed at $450 \mathrm{~nm}$ with a microplate reader (BioTech Instruments, Winooski, VT, USA) at 24-h intervals for 5 continuous days.
Colony formation assay. After transfection with miR-15a or $\mathrm{NC}$ for $48 \mathrm{~h}, 769-\mathrm{P}$ and OS-RC-2 cells were trypsinized and replaced into 6-well plates for colony formation assay. After 5 days, the cells were fixed in $4 \%$ formaldehyde, stained with crystal violet, and the number of colonies ( $>50$ cells) were counted.

Matrigel invasion assay. Cell invasion assay was performed using a Transwell chamber $(8-\mu \mathrm{m}$ pore size $)$. After transfection with miR-15a mimics or NC for $48 \mathrm{~h}, 769-\mathrm{P}$ and OS-RC-2 cells were diluted in serum-free DMEM and plated in the top chamber, which was pre-coated with Matrigel (BD Biosciences, San Jose, CA, USA) to assess cell invasion. Meanwhile, DMEM containing $10 \%$ fetal bovine serum (FBS) was added to the bottom chamber as the chemoattractant. After incubation for $48 \mathrm{~h}$, the migrated cells on the bottom chamber were fixed in $4 \%$ paraformaldehyde and stained with crystal violet, and counted under an inverted microscope (Olympus, Tokyo, Japan).

Cell cycle assay. After transfection with the miR-15a mimics or NC for $36 \mathrm{~h}, 769-\mathrm{P}$ and OS-RC-2 cells were trypsinized and collected. For cell cycle analysis, the cells were fixed with ice-cold ethanol at $4^{\circ} \mathrm{C}$ overnight. Then, the cells were centrifuged at $1,000 \mathrm{rpm}$ for $5 \mathrm{~min}$ at $4^{\circ} \mathrm{C}$, and the pellets were treated with $50 \mu \mathrm{g} / \mathrm{ml}$ propidium iodide (PI) and $100 \mu \mathrm{g} / \mathrm{ml}$ RNase A at room temperature in the dark for $30 \mathrm{~min}$. Cell cycle distribution was then analyzed using a flow cytometric system (FACS; BD Biosciences).

Cell apoptosis assay. Cell apoptosis was analyzed using Annexin V/PI apoptosis detection kit (Beyotime, Shanghai, China) according to the manufacturer's instructions. After transfection with the miR-15a mimics or NC for $36 \mathrm{~h}, 769-\mathrm{P}$ and OS-RC-2 cells were trypsinized and centrifuged at 1,000 rpm for $5 \mathrm{~min}$, and then resuspended with $1 \mathrm{X}$ binding buffer, the cells were then incubated with $1 \mu \mathrm{l}$ Annexin V-FITC and $5 \mu \mathrm{l}$ $\mathrm{PI}$ at room temperature in the dark for $5 \mathrm{~min}$. Cell apoptosis was assessed by flow cytometry (FACS).

Western blotting. After $36 \mathrm{~h}$ transfection, 769-P and OS-RC-2 cells were treated with RIPA lysis buffer for $40 \mathrm{~min}$ on ice. The protein concentration was determined using a BCA protein assay kit (Bio-Rad Laboratories, Hercules, CA, USA), as described in the manufacturer's manual. Lysate proteins $(40 \mu \mathrm{g})$ were subjected to $10 \%$ SDS-PAGE and electrophoretically transferred to polyvinylidene difluoride (PVDF) membranes. After blotting, the membranes were blocked with 5\% fat-free dry milk for $1 \mathrm{~h}$ and then incubated with the specific primary antibody against eIF4E, cyclin D1, c-Myc, MMP3, Bcl-2, P13K, p-AKT, mTOR or $\beta$-actin (Santa Cruz Biotechnology, Santa Cruz, CA, USA) for $2 \mathrm{~h}$ at $37^{\circ} \mathrm{C}$. After rinsing, membranes were hybridized with HRP-conjugated secondary antibodies for $1 \mathrm{~h}$ at $37^{\circ} \mathrm{C}$. The protein bands were detected using a quantitative gel and a Western Blot Imaging System (FluorChem Q; Alpha Innotech, San Leandro, CA, USA).

RNA isolation and quantitative real-time PCR. Total RNA was extracted from tissue samples and cell lines using TRIzol (Invitrogen) according to the manufactuer's protocol. 

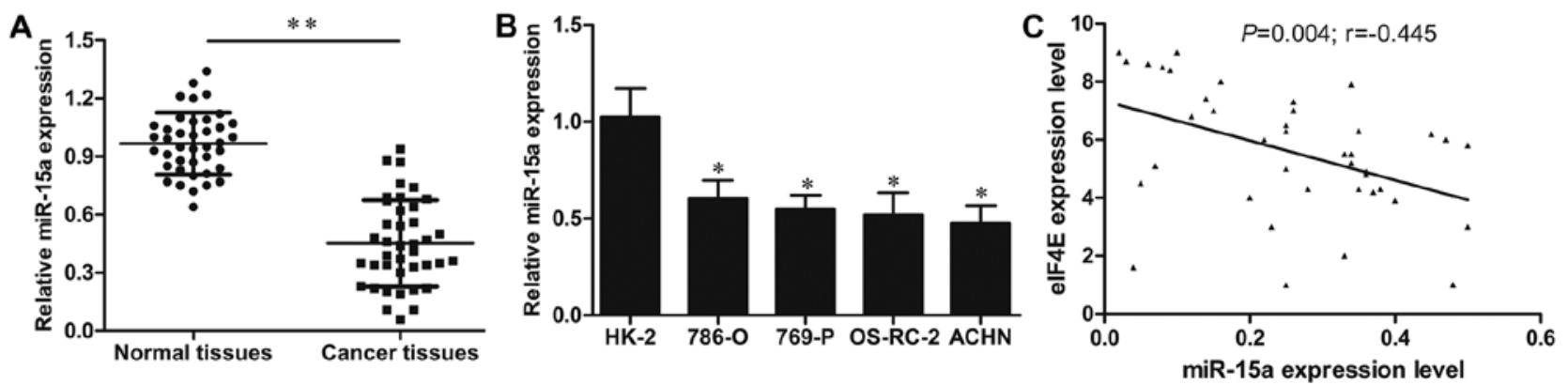

Figure 1. Expression of miR-15a is downregulated in RCC tissues and cell lines. (A) qRT-PCR detection of miR-15a expression in 40 RCC specimens and adjacent normal tissues. (B) qRT-PCR detection of miR-15a expression in 4 RCC cell lines and normal renal cell line HK-2. (C) Correlation between miR-15a and eIF4E mRNA in RCC tissues using linear correlation. Each experiment was repeated at least 3 times; ${ }^{*} \mathrm{P}<0.05$, ${ }^{* *} \mathrm{P}<0.01$ vs. the control group.

RNA was reversely transcribed into cDNA using a Reverse Transcirption kit (Takara, Dalian, China). Quantitative real-time PCR (qRT-PCR) analysis was performed using a SYBR-Green PCR Master Mix (Takara) on an ABI 7900HT PCR System (Applied Biosystems, Foster City, CA, USA). The special primers used for miR-15a, eIF4E, U6 and $\beta$-actin were synthesized from GenScript Co., Ltd. (Nanjing, China). The relative expression levels of the genes were quantified using the $2^{-\Delta \Delta C t}$ method. U6 and $\beta$-actin were used as the endogenous controls for normalization.

Bioinformatics and luciferase assay. An online miRNA database (http://www.microrna.org/microrna/home.do) was used to predict the potential target gene binding site of miR-15a. The wild-type and mutant pGL3-eIF4E 3'-UTR were constructed and co-transfected with the miR-15a mimics or NC into 769-P and OS-RC-2 cells using Lipofectamine 2000 (Invitrogen) according to the manufacturer's instructions. After $36 \mathrm{~h}$ of transfection, the luciferase reporter activity was assesed using the Renilla-Firefly Dual-Luciferase Assay kit (Thermo Fisher Scientific, Inc., Waltham, MA, USA) according to the manufacturer's protocol. Firefly luciferase activity was normalized to Renilla luciferase activity.

Immunohistological staining. All 40-paired fresh specimens were fixed with $10 \%$ formalin and embedded in paraffin using the standard protocol. The slides were then deparaffinized in xylene and subsequently rehydrated by a graded ethanol series ending in deionized water. Any endogenous peroxidase was blocked by treatment with $3 \%$ hydrogen peroxide for $15 \mathrm{~min}$, followed by 3 rinses of 5 min each in deionized water. Antigen retrieval was performed by placing slides in $1 \mathrm{X}$ citrate buffer for $15 \mathrm{~min}$ at $100^{\circ} \mathrm{C}$ (in a microwave oven). The rabbit antihuman eIF4E primary antibody (diluted 1:150; Cell Signaling Technology Inc., Beverly, MA, USA) was left on tissue samples overnight at $4^{\circ} \mathrm{C}$ in a humidified chamber. The secondary antibody (ZSGB-BIO Co., Ltd., Beijing, China) was then applied to all sections and incubated for $30 \mathrm{~min}$ at room temperature. Finally, the sections were developed with 3,3'-diaminobenzidine for $5 \mathrm{~min}$ at room temperature, and then counterstained with hematoxylin. After staining, the sections were dehydrated through a graded series of ethanol washes, cleared in xylene and cover-slipped. Positive reactivity was detected with a diaminobenzidine kit (ZSGB-BIO Co., Ltd.).
Statistical analysis. Where necessary, data are expressed as the means \pm SD, and one-way ANOVA coupled with a Student's t-test were performed to compare differences between experimental groups using SPSS 11.5 statistical software (SPSS, Inc., Chicago, IL, USA). Spearman's correlation analysis was applied to assess the correlation between eIF4E expression and miR-15a in RCC tissues. Each experiment was repeated at least 3 times. The criterion for statistical significance was denoted as $\mathrm{P}<0.05$.

\section{Results}

miR-15a is downregulated in human RCC tissues. qRT-PCR method was performed to investigate the expression levels of miR-15a in 40-paired cases of RCC and adjacent normal tissues. As shown in Fig. 1A, the expression levels of miR-15a were significantly decreased in RCC tissues compared to those in adjacent matched normal tissues $(\mathrm{P}<0.01)$. Furthermore, the expression of miR-15a was also detected in 4 human RCC cell lines ACHN, 786-O, 769-P, OS-RC-2 and one normal renal cell line $\mathrm{HK}-2$. The results revealed that the expression level of miR-15a in ACHN, 786-O, 769-P and OS-RC-2 cells was markedly decreased when compared to that in the HK-2 cell line (all $\mathrm{P}<0.05$; Fig. 1B). These findings revealed that miR-15a may be involved in RCC progression.

miR-15a suppresses RCC cell proliferation and invasion. To determine the effect of miR-15a in RCC development, miR-15a mimics were transfected into the RCC cell lines 769-P and OS-RC-2 for $48 \mathrm{~h}$, which exhibited a high transfection efficiency ( $\mathrm{P}<0.01$, respectively; Fig. $2 \mathrm{~A})$. A CCK-8 assay revealed that miR-15a significantly suppressed the cell growth rate of 769-P and OS-RC-2 cells during the 5 days of detection (Fig. 2B). The colony formation assay also revealed that the colony formation abilities of 769-P and OS-RC-2 cells transfected with the miR-15a mimics were significantly decreased compared to those in the $\mathrm{NC}$ groups $(\mathrm{P}<0.01$, respectively; Fig. 2C). In addition, a Matrigel invasion assay demonstrated that overexpression of miR-15a markedly decreased the number of invasive cells for 769-P and OS-RC-2 cells in comparison to the NC groups ( $\mathrm{P}<0.01$, respectively; Fig. 2D).

miR-15a induces apoptosis and causes G0/G1 cell cycle arrest in RCC cells. The cell cycle distribution in 769-P and OS-RC-2 

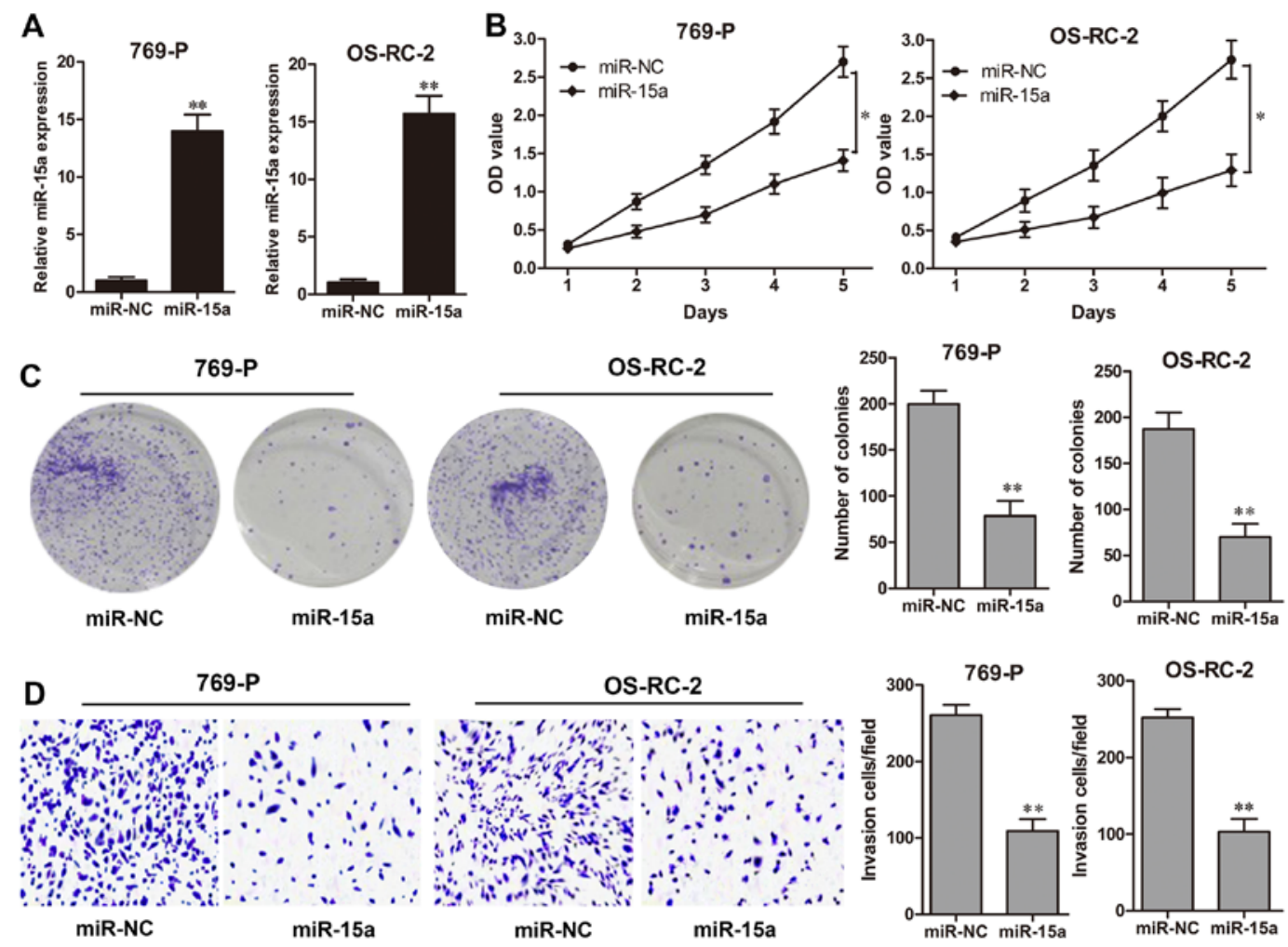

Figure 2. Effects of miR-15a on proliferation and invasion in RCC cells. (A) The efficiency of miR-15a-mimic transfection in 769-P and OS-RC-2 cells was detected using qRT-PCR. (B) A CCK-8 assay revealed that miR-15a mimics inhibited the growth rate of 769-P and OS-RC-2 cells transfected with the mimics. (C) The number of colonies in 769-P and OS-RC-2 cells transfected with the miR-15a mimics was analyzed by crystal violet staining. (D) Invasive ability of 769-P and OS-RC-2 cells transfected with the miR-15a mimics was assessed by Matrigel invasion assays. Each experiment was repeated at least 3 times; ${ }^{*} \mathrm{P}<0.05,{ }^{* *} \mathrm{P}<0.01$ vs. the $\mathrm{NC}$ group.

cells after miR-15a-mimic transfection was determined using flow cytometry. As shown in Fig. 3A, miR-15a overexpression markedly increased the proportion of G1/G0-phase cells in the 769-P and OS-RC-2 cells, when compared with that of the $\mathrm{NC}$ groups $(\mathrm{P}<0.05$, respectively); while it decreased the percentage of S-phase cells in the 769-P and OS-RC-2 cells compared with that of the $\mathrm{NC}$ groups $(\mathrm{P}<0.05$, respectively). These findings indicated that miR-15a overexpression could cause cell G1/G0-phase arrest and result in the decrease of G1 to $\mathrm{S}$ phase transition. Furthermore, to evaluate the function of miR-15a on RCC cell apoptosis, a flow cytometric assay was also performed using Annexin V and PI staining. The results revealed that transfection of 769-P and OS-RC-2 cells with miR-15a mimics markedly induced cell apoptosis in comparison to the $\mathrm{NC}$ groups $(\mathrm{P}<0.01$; respectively, Fig. 3B), indicating that miR-15a promoted apoptosis in RCC cells.

To further validate the effect of miR-15a on RCC cell proliferation, apoptosis and invasion-related proteins, the expression levels of cyclin D1, c-Myc, Bcl-2, MMP3 were detected in 769-P and OS-RC-2 cells with miR-15a-mimic transfection. Western blot assay revealed that overepxression of miR-15a downregulated the expression of cyclin D1, cyclin E, Bax, c-Myc and MMP3 (all $\mathrm{P}<0.05$ ), but upregulated the expression of $\mathrm{Bcl} 2(\mathrm{P}<0.05$; Fig. $3 \mathrm{C})$ significantly. In addition, the $\mathrm{P} 13 \mathrm{~K} / \mathrm{AKT} / \mathrm{mTOR}$ signaling pathway was also detected, and the results revealed that the expression levels of P13K, mTOR and phosphorylated (p)-AKT were significantly decreased in 769-P and OS-RC-2 cells with miR-15a-mimic transfection compared to those in the NC groups (all $\mathrm{P}<0.05$; Fig. 3C).
eIF4E serves as a target of miR-15a. Using bioinformatic analysis (microRNAs database), we found that the 3'-UTR of eIF4E possessed a putative target for miR-15a (Fig. 4A). A luciferase reporter assay was applied to determine the functional binding site of miR-15a. The results revealed that the luciferase reporter activity was significantly decreased in 769-P and OS-RC-2 cells co-transfected with the pGL3-eIF4Ewild-type 3'-UTR and miR-15a mimics, when compared with those co-tranfected with $\mathrm{NC}(\mathrm{P}<0.05$, Fig. 4B). However, no significant change was detected in 769-P and OS-RC-2 cells co-transfected with pGL3-eIF4E-mutant construct (Fig. 4B). Moreover, after transfection 769-P and OS-RC-2 cells with miR-15a or NC, qRT-PCR and western blot analysis showed that eIF4E expression was significantly decreased in the miR-15a-mimic groups compared with that of the NC groups at both the mRNA and protein levels $(\mathrm{P}<0.05$, respectively; Fig. 4C). These results revealed that miR-15a may directly bind to the 3'-UTR region of eIF4E and suppress eIF4E expression. To validate this hypothesis, we then examined the eIF4E expression levels in 40 cases of RCC by immunohistochemical staining and qRT-PCR assays. The results from immunohistochemical staining revealed that eIF4E was barely detectable in normal renal tissues, while it exhibited positive nuclear immunostaining in 26 out of 40 cancer tissues (Fig. 4D). Furthermore, the correlation between miR-15a and eIF4E mRNA expression in 40 cases of RCC tissues was assessed by Spearman's correlation analysis. As shown in Fig. 1C, a significant negative correlation was observed between the mRNA levels of eIF4E and miR-15a expression $(r=-0.445 ; \mathrm{P}<0.01)$. 

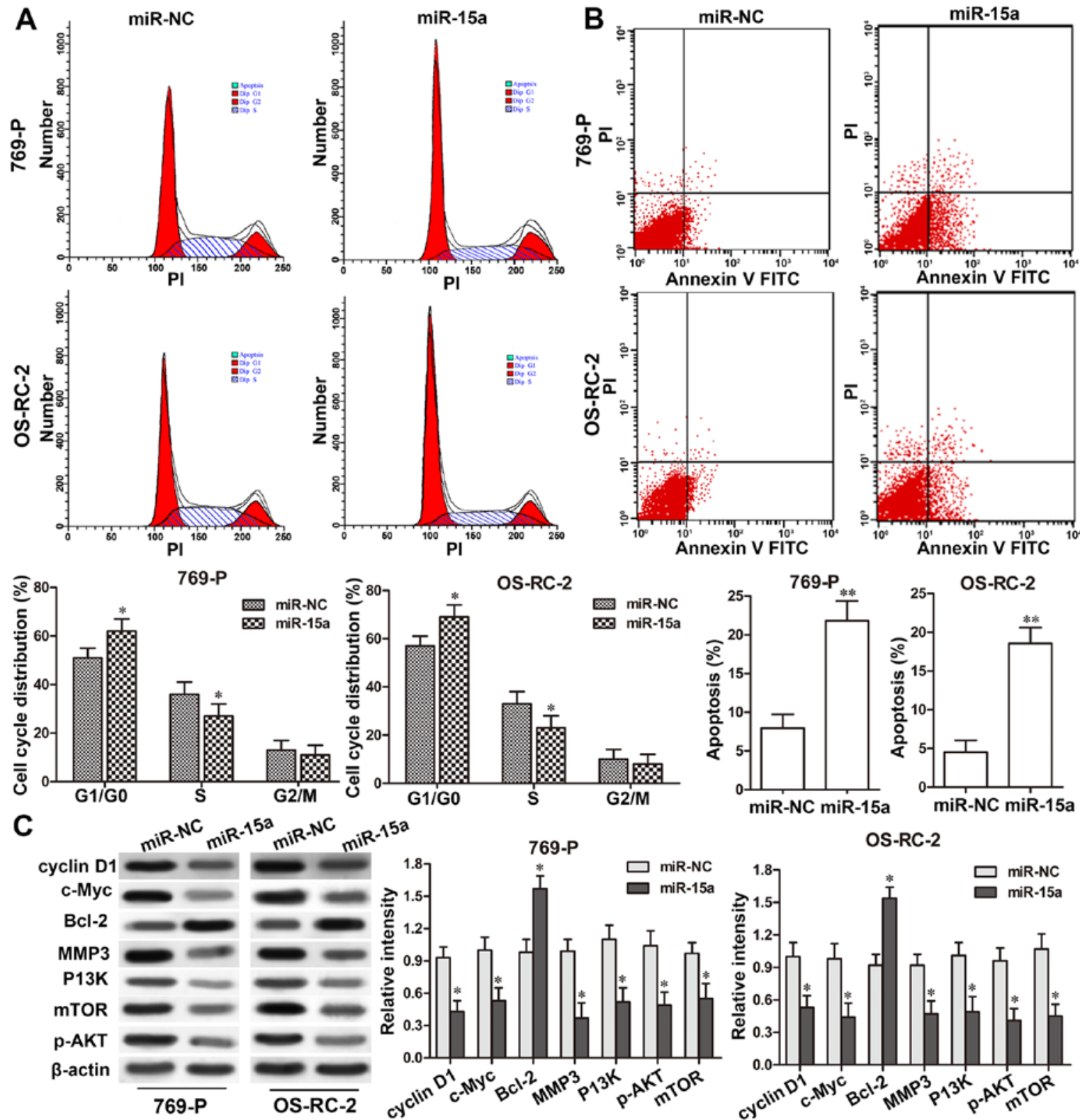

OS-RC-2

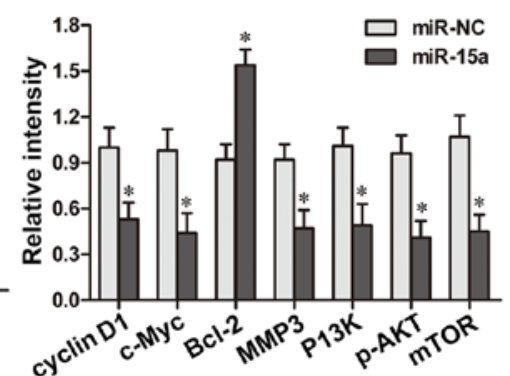

Figure 3. Influence of miR-15a on the cell cycle and apoptosis of RCC cells. (A) Cell cycle distribution of 769-P and OS-RC-2 cells with miR-15a transfection was assessed by flow cytometry. (B) Cell apoptosis of 769-P and OS-RC-2 cells with miR-15a transfection was stained with Annexin V and PI and analyzed by flow cytometry. (C) The expression levels of proliferation- and invasion-related proteins in 769-P and OS-RC-2 cells with miR-15a transfection were detected by western blotting. Each experiment was repeated at least 3 times; ${ }^{*} \mathrm{P}<0.05,{ }^{* *} \mathrm{P}<0.01$ vs. the $\mathrm{NC}$ group.

eIF4E is involved in the regulation of RCC cell proliferation and invasion by $m i R-15$. A rescue experiment was performed to further determine that miR-15a suppressed the proliferation and invasion of RCC cells by directly regulating eIF4E. As shown in Fig. 5A, eIF4E was significantly overexpressed in 769-P and OS-RC-2 cells with eIF4E-plasmid transfection $(\mathrm{P}<0.01$, respectively). A CCK-8 assay revealed that the cell growth rate was significantly enhanced in the 769-P and OS-RC-2 cells with eIF4E overexpression, and partially reversed the suppression of cell growth resulted from the miR-15a mimics (Fig. 5B). Furthermore, a Matrigel invasion assay also illustrated that overexpression of eIF4E significantly promoted cell invasive abilities in 769-P and OS-RC-2 cells ( $\mathrm{P}<0.01$, respectively), and eIF4E markedly abolished the inhibition of cell invasion caused by the miR-15a mimics $(\mathrm{P}<0.01$ vs. the vector + miR-15a, respectively; Fig. 5C). Furthermore, the western blot results also revealed that overexpression of eIF4E significantly upregulated the expression of P13K, mTOR and p-AKT (all $\mathrm{P}<0.05$ ), and blocked the influence of miR-15a mimics on the activation of the $\mathrm{P} 13 \mathrm{~K} / \mathrm{AKT} / \mathrm{mTOR}$ signaling pathway (all $\mathrm{P}<0.05$ vs. the vector + miR-15a; Fig. 5D). Collectively, these findings demonstrated that eIF4E was involved in miR-15a-induced inhibition of cell proliferation and invasion of RCC cells which may be mediating by the activation of the $\mathrm{P} 13 \mathrm{~K} / \mathrm{AKT} / \mathrm{mTOR}$ signaling pathway.

\section{Discussion}

Since RCC ususally presents with metastasis at the time of diagnosis in adults, identifying valuable epigenetic biomarkers that could better improve the accuracy of diagnosis and treatment are urgently needed (20). Increasing evidence 

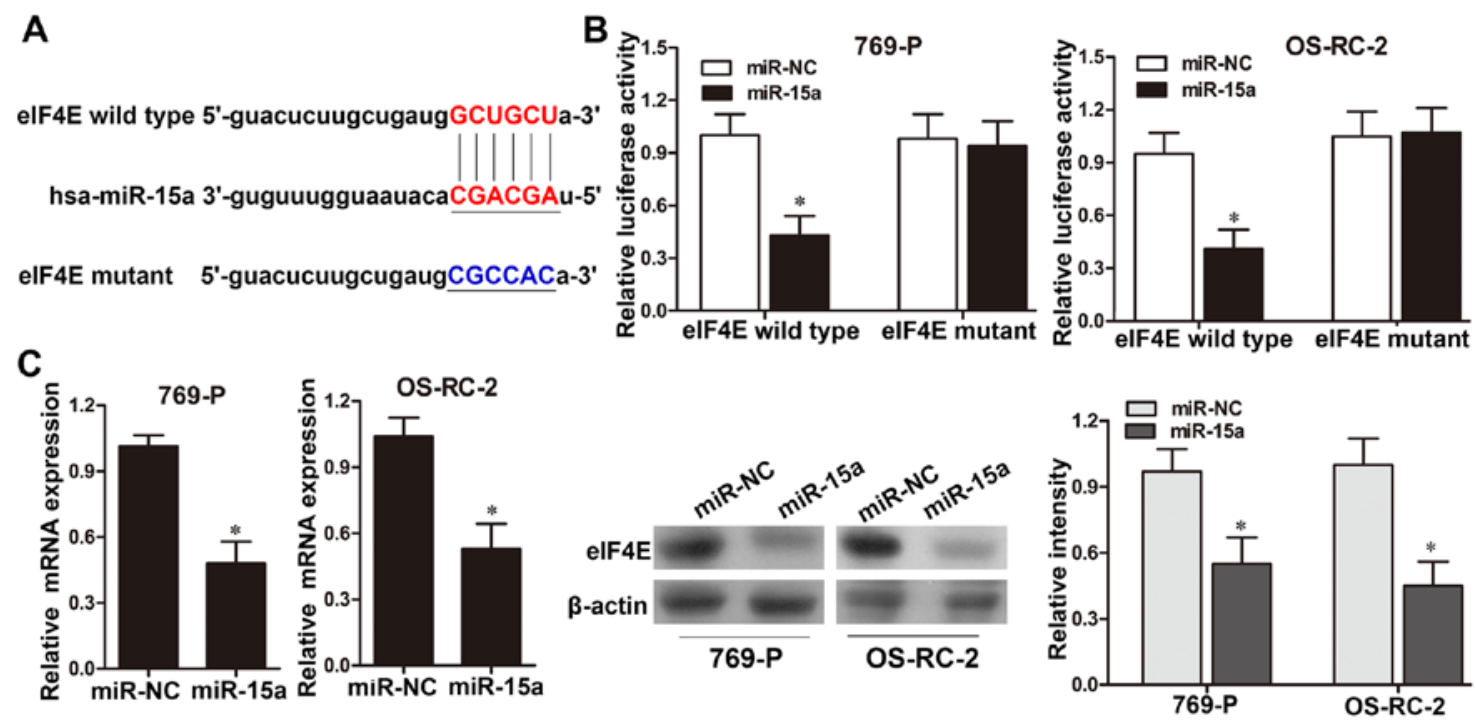

D
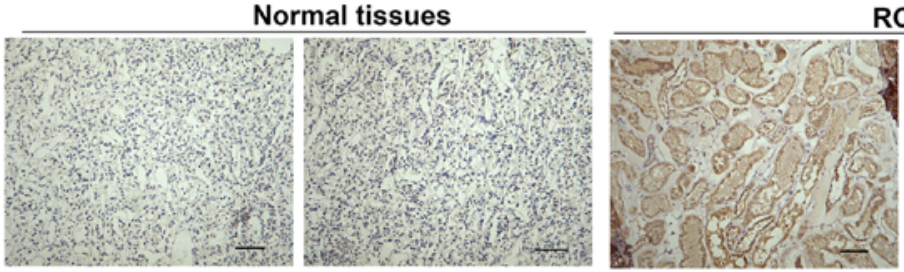

RCC tissues

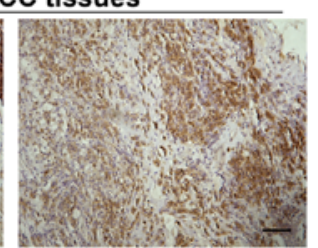

Figure 4. miR-15a directly targets eIF4E in RCC cells. (A) The binding site of miR-15a in the 3'-UTR of eIF4E was predicted by bioinformatics analysis. (B) The relative luciferase activity was assessed in 769-P and OS-RC-2 cells after co-transfection of the pGL3-eIF4E wild-type or mutant construct with miR-15a mimics or NC. (C) The expression levels of eIF4E in 769-P and OS-RC-2 cells with miR-15a transfection were determined by qRT-PCR and western blotting. (D) The expression and distribution of eIF4E in clinical RCC specimens was detected by immunohistochemical staining (magnification, $\mathrm{x} 400$ ). Each experiment was repeated at least 3 times. Scale bar, $50 \mu \mathrm{m} ;{ }^{*} \mathrm{P}<0.05$ vs. the $\mathrm{NC}$ group.

has demonstrated that aberrant expression of various miRs contribute to the pathogenesis and tumorigenesis of cancers through their participation in many cellular processes, including cell proliferation, invasion and angiogenesis (21). In the present study, we revealed that miR-15a was significantly downregulated in RCC tissues and cell lines. Ectopic expression of miR-15a suppressed RCC cell proliferation and invasion in vitro. Furthermore, eIF4E was identified as a direct and functional target of miR-15a and partially reversed the miR-15a-induced suppression of RCC cell proliferation and invasion. These data strongly revealed a possible tumorsuppressor role for miR-15a in RCC development.

The downregualtion of miR-15a has been reported to be associated with cell proliferation and metastases in several types of cancer. Yang et al revealed that miR-15a acted as a tumor suppressor in non-small cell lung cancer by inducing cell apoptosis and inhibiting metastasis (12). Alderman et al found that miR-15a inhibited the proliferation and invasiveness of melanoma cells by directly targeting the CDCA4 gene (13). $\mathrm{Li}$ et al demonstrated that ectopic expression of miR-15a regulated cell cycle arrest at the G1 phase and potentiated apoptosis in breast cancer cells (22). Consistent with the previous studies, using CCK-8 proliferation and colony formation assays we found that overexpresison of miR-15a significantly inhibited the cell growth of RCC cells. Furthermore, the flow cytometric assay confirmed that miR-15a mimic arrested RCC cell cycle by inhibiting the G1 and S phase transition and induced cell apoptosis. Supplementary evidence also revealed the inhibitory effect of miR-15a on RCC cell metastases. Consequently, these findings demonstrated that miR-15a served as a tumor suppressor in the progression of RCC cells, and may be used as a potential therapeutic biomarker for RCC treatment.

Previously, aberrant upregulation of eIF4E has been found in many tumors, such as head and neck squamous cell carcinoma, lung cancer, breast cancer, and thyroid cancer (23). Silencing of eIF4E revealed cell proliferation and arrested the cell cycle at the G0/G1 phase, and inhibition of eIF4E could decrease the invasion of cancer cells (24). Furthermore, accumulating studies reveal that overexpression of eIF4E is sufficient to abrogate apoptosis and lead to chemoresistance and radio-resistance in cancer cells $(25,26)$. Recent studies reveal evidence that eIF4E is maintained at levels in excess for normal development that are hijacked by cancer cells to drive a translational program supporting tumorigenesis, such as proliferation and survival-promoting associated proteins cyclin D1, c-Myc, vascular endothelial growth factor (VEGF), survivin and Bcl-2 (27,28). These findings indicate that targeting the expression of eIF4E may provide a potential molecular target for cancer therapy. For example, eIF4E can be suppressed by some miRNAs. Li et al demonstrated that microRNA-497 modulated gastric cancer cell proliferation and invasion by suppressing eIF4E (29). Liu et al also pointed out that miR-34c-3p functioned as a tumor suppressor by inhibiting eIF4E in non-small cell lung cancer (30).

In the present study, bioinformatic analysis determined eIF4E to be a putative target for miR-15a. The luciferase assay using a reporter containing the wild-type miR-15a binding sequence at the 3'-UTR of eIF4E mRNA indicated 

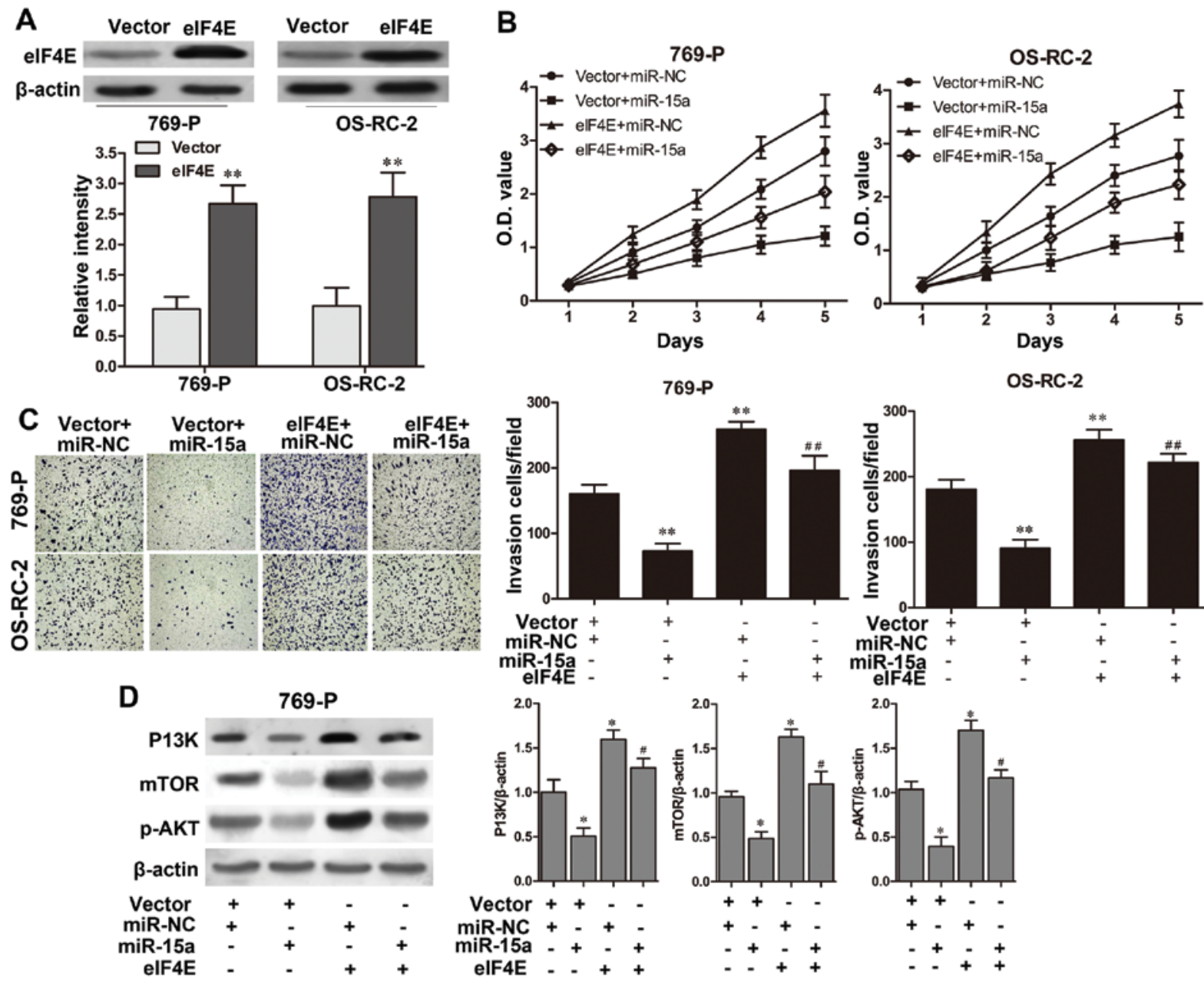

OS-RC-2
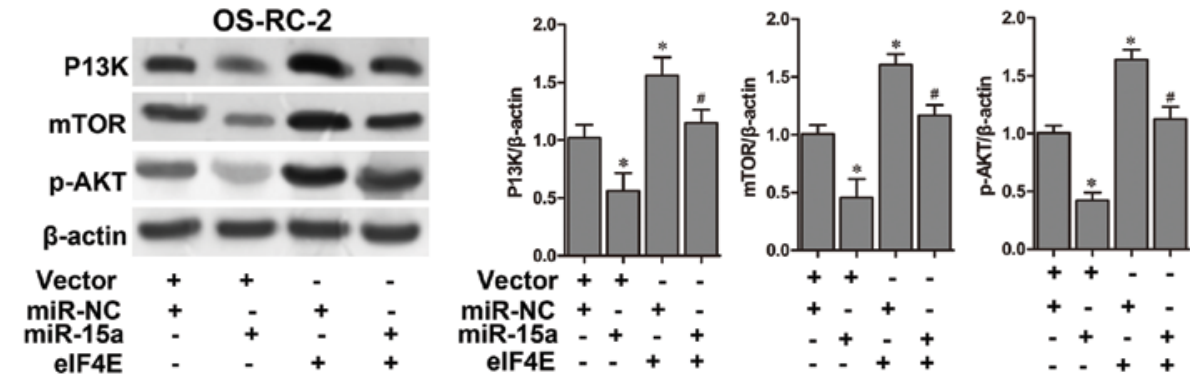

Figure 5. eIF4E is involved in mediating RCC cell growth and invasion by miR-15a. (A) The expression of eIF4E was assessed by western blotting. (B) The inhibitory growth rate and (C) invasive ability of 769-P and OS-RC-2 cells with miR-15a transfection was partially reversed by eIF4E overexpression. (D) The effect of eIF4E overexpression on the activation of P13K/AKT/mTOR signaling was assessed by western blotting. Each experiment was repeated at least 3 times; ${ }^{*} \mathrm{P}<0.05,{ }^{* *} \mathrm{P}<0.01$ vs. the NC group; ${ }^{*} \mathrm{P}<0.05,{ }^{\# \#} \mathrm{P}<0.01$ vs. the vector $+\mathrm{miR}-15$ a group.

that the luciferase activity could be significantly decreased by overexpression of miR-15a. eIF4E participates in nuclear mRNA export and translation of specific transcripts, which are necessary for the promotion of cell proliferation, survival and metastases. In the present study, eIF4E was found to be both overexpressed and highly enriched in the nucleus of these specimens. Endogenous expression of miR-15a could downregulate the expression of eIF4E mRNA and protein levels in RCC cells. Furthermore, miR-15a levels were found to be inversely correlated with mRNA expression of eIF4E in RCC tissues, suggesting that miR-15a suppressed RCC development by directly targeting endogenous eIF4E.

In the present study, ectopic expression of eIF4E in RCC cells could partially rescue the inhibitory effect of miR-15a overexpression on cell proliferation and invasion. Furthermore, ectopic expression of eIF4E activated the PI3K/Akt/mTOR signaling pathway, which was suppressed by miR-15a. It has also been demonstrated that eIF4E is the crossing point of the PI3K/Akt/mTOR signaling pathway and the Ras/Raf/MEK/ ERK/Mnks signaling pathway $(15,31)$. The phosphoinositide 3-kinase/ protein kinase $\mathrm{B} /$ mammalian target of rapamycin (P13K/AKT/mTOR) is a crucial and intensively explored intracellular signaling pathway in tumorigenesis (32). PI3K signaling leads to phosphorylation of Akt and the activation of protein translation initiation via mTOR (upstream and downstream of mTOR), a phenomenon which has been widely investigated in various types of cancer and other aggressive diseases $(33,34)$. Activation of the P13K/AKT/mTOR 
signaling pathway mediated through molecular aberrations such as eIF4E is instrumental in promoting RCC development including cell proliferation, apoptosis and migration as well as resistance to anticancer therapies $(35,36)$. Consequently, in the present study, we surmised that miR-15a activated the P13K/ AKT/mTOR signaling pathway and it downstream factors through binding to eIF4E in RCC cells.

In summary, miR-15a expression was found to be downregulated in RCC clinical specimens and cell lines. Overexpression of miR-15a played a crucial role in suppressing cell growth and invasion, causing cell cycle arrest and inducing cell apoptosis in the progression of RCC by directly targeting eIF4E, thus suggesting the potential of miR-15a to function as a tumor suppressor and a therapeutic biomarker in RCC development.

\section{Acknowledgements}

The present study was supported by the Science and Technique Project of Xi'an [SF1418 (1)].

\section{References}

1. Chen W, Zheng R, Baade PD, Zhang S, Zeng H, Bray F, Jemal A, Yu XQ and He J: Cancer statistics in China, 2015. CA Cancer J Clin 66: 115-132, 2016.

2. Siegel RL, Miller KD and Jemal A: Cancer Statistics, 2017. CA Cancer J Clin 67: 7-30, 2017.

3. Buonerba C, Di Lorenzo G and Sonpavde G: Combination therapy for metastatic renal cell carcinoma. Ann Transl Med 4 $100,2016$.

4. Escudier B: Advanced renal cell carcinoma: Current and emerging management strategies. Drugs 67: 1257-1264, 2007.

5. Afriansyah A, Hamid AR, Mochtar CA and Umbas R: Targeted Therapy for Metastatic Renal Cell Carcinoma. Acta Med Indones 48: 335-347, 2016.

6. Hammond SM: An overview of microRNAs. Adv Drug Deliv Rev 87: 3-14, 2015.

7. Simonson B and Das S: MicroRNA therapeutics: The next magic bullet? Mini Rev Med Chem 15: 467-474, 2015.

8. Shah MY, Ferrajoli A, Sood AK, Lopez-Berestein G and Calin GA: MicroRNA therapeutics in cancer - an emerging concept. EBioMedicine 12: 34-42, 2016.

9. Acunzo M, Romano G, Wernicke D and Croce CM: MicroRNA and cancer - a brief overview. Adv Biol Regul 57: 1-9, 2015.

10. Li X, Xin S, He Z, Che X, Wang J, Xiao X, Chen J and Song X: MicroRNA-21 (miR-21) post-transcriptionally downregulates tumor suppressor PDCD4 and promotes cell transformation proliferation, and metastasis in renal cell carcinoma. Cell Physiol Biochem 33: 1631-1642, 2014.

11. Xu M, Gu M, Zhang K, Zhou J, Wang Z and Da J: miR-203 inhibition of renal cancer cell proliferation, migration and invasion by targeting of FGF2. Diagn Pathol 10: 24, 2015.

12. Yang T, Thakur A, Chen T, Yang L, Lei G, Liang Y, Zhang S, Ren $\mathrm{H}$ and Chen M: MicroRNA-15a induces cell apoptosis and inhibits metastasis by targeting BCL2L2 in non-small cell lung cancer. Tumour Biol 36: 4357-4365, 2015.

13. Alderman C, Sehlaoui A, Xiao Z and Yang Y: MicroRNA-15a inhibits the growth and invasiveness of malignant melanoma and directly targets on CDCA4 gene. Tumour Biol 37: 13941-13950, 2016.

14. Luo Q, Li X, Li J, Kong X, Zhang J, Chen L, Huang Y and Fang L: MiR-15a is underexpressed and inhibits the cell cycle by targeting CCNE1 in breast cancer. Int J Oncol 43: 1212-1218, 2013.
15. Siddiqui N and Sonenberg N: Signalling to eIF4E in cancer. Biochem Soc Trans 43: 763-772, 2015.

16. Sonenberg N: eIF4E, the mRNA cap-binding protein: From basic discovery to translational research. Biochem Cell Biol 86: 178-183, 2008.

17. Jia Y, Polunovsky V, Bitterman PB and Wagner CR: Cap-dependent translation initiation factor eIF4E: An emerging anticancer drug target. Med Res Rev 32: 786-814, 2012.

18. Truitt ML, Conn CS, Shi Z, Pang X, Tokuyasu T, Coady AM, Seo Y, Barna M and Ruggero D: Differential requirements for eIF4E dose in normal development and cancer. Cell 162: 59-71, 2015.

19. Campbell L, Jasani B, Griffiths DF and Gumbleton M: Phospho-4e-BP1 and eIF4E overexpression synergistically drives disease progression in clinically confined clear cell renal cell carcinoma. Am J Cancer Res 5: 2838-2848, 2015.

20. Stewart GD, O'Mahony FC, Powles T, Riddick ACP, Harrison DJ and Faratian D: What can molecular pathology contribute to the management of renal cell carcinoma? Nat Rev Urol 8: 255-265, 2011.

21. Di Leva G, Garofalo M and Croce CM: MicroRNAs in cancer. Annu Rev Pathol 9: 287-314, 2014.

22. Li P, Xie XB, Chen Q, Pang GL, Luo W, Tu JC, Zheng F, Liu SM, Han L, Zhang J-K, et al: MiRNA-15a mediates cell cycle arrest and potentiates apoptosis in breast cancer cells by targeting synuclein- $\gamma$. Asian Pac J Cancer Prev 15: 6949-6954, 2014.

23. Graff JR, Konicek BW, Carter JH and Marcusson EG: Targeting the eukaryotic translation initiation factor $4 \mathrm{E}$ for cancer therapy. Cancer Res 68: 631-634, 2008

24. De Benedetti A and Graff JR: eIF-4E expression and its role in malignancies and metastases. Oncogene 23: 3189-3199, 2004.

25. Wan J, Shi F, Xu Z and Zhao M: Knockdown of eIF4E suppresses cell proliferation, invasion and enhances cisplatin cytotoxicity in human ovarian cancer cells. Int J Oncol 47: 2217-2225, 2015.

26. Li Y, Fan S, Koo J, Yue P, Chen ZG, Owonikoko TK, Ramalingam SS, Khuri FR and Sun SY: Elevated expression of eukaryotic translation initiation factor $4 \mathrm{E}$ is associated with proliferation, invasion and acquired resistance to erlotinib in lung cancer. Cancer Biol Ther 13: 272-280, 2012.

27. Martineau Y, Azar R, Bousquet C and Pyronnet S: Anti-oncogenic potential of the eIF4E-binding proteins. Oncogene 32: 671-677, 2013.

28. Hsieh AC and Ruggero D: Targeting eukaryotic translation initiation factor 4E (eIF4E) in cancer. Clin Cancer Res 16: 49144920, 2010

29. Li W, Jin X, Deng X, Zhang G, Zhang B and Ma L: The putative tumor suppressor microRNA-497 modulates gastric cancer cell proliferation and invasion by repressing eIF4E. Biochem Biophys Res Commun 449: 235-240, 2014.

30. Liu F, Wang X, Li J, Gu K, Lv L, Zhang S, Che D, Cao J, Jin S and Yu Y: miR-34c-3p functions as a tumour suppressor by inhibiting eIF4E expression in non-small cell lung cancer. Cell Prolif 48: 582-592, 2015

31. Raught B and Gingras AC: eIF4E activity is regulated at multiple levels. Int J Biochem Cell Biol 31: 43-57, 1999.

32. Polivka J Jr and Janku F: Molecular targets for cancer therapy in the PI3K/AKT/mTOR pathway. Pharmacol Ther 142: 164-175, 2014.

33. Zhang X, Shi H, Tang H, Fang Z, Wang J and Cui S: miR-218 inhibits the invasion and migration of colon cancer cells by targeting the PI3K/Akt/mTOR signaling pathway. Int J Mol Med 35: 1301-1308, 2015.

34. Dancey JE: Therapeutic targets: MTOR and related pathways. Cancer Biol Ther 5: 1065-1073, 2006.

35. Sun SY, Rosenberg LM, Wang X, Zhou Z, Yue P, Fu H and Khuri FR: Activation of Akt and eIF4E survival pathways by rapamycin-mediated mammalian target of rapamycin inhibition. Cancer Res 65: 7052-7058, 2005.

36. Ilic N, Utermark T, Widlund HR and Roberts TM: PI3K-targeted therapy can be evaded by gene amplification along the MYC-eukaryotic translation initiation factor 4E (eIF4E) axis. Proc Natl Acad Sci USA 108: E699-E708, 2011. 